\title{
Gamification of Geographic Data Collection
}

\author{
Dražen ODOBAŠIĆ ${ }^{1}$, Damir MEDAK ${ }^{1}$ and Mario MILER ${ }^{1}$ \\ ${ }^{1}$ Faculty of Geodesy, University of Zagreb/Croatia · dodobas@geof.hr
}

This contribution was double-blind reviewed as full paper.

\begin{abstract}
Using game design elements in non-game contexts is an established concept. Gamification is all about improving actual user engagement with the system, making users contribute more time and resources than they have intended, by rewarding them with status, access, power and stuff (SAPS). In this review paper we give an overview of gamification, and user motivations for playing games, and we discuss basic game design elements. We also compare existing services, like Foursquare, Waze and Ingress that use game design elements in geographic data collection processes. Services are compared in the context of the SAPS model and actual used game mechanics, including points, levels, leaderboards, badges and challenges. Gamification can be applied to geographic data collection processes as shown by the services analysed in this article. Current research of gamification applied to geographic data collection gives no definite conclusions. We plan to focus our further research on gamification in the context of geographic data collection, particularly in the realm of volunteered geographic information.
\end{abstract}

\section{Introduction}

The main objective of this paper is to give an overview of gamification as it applies to geographic data collection. A game is "structured playing, usually undertaken for enjoyment and sometimes used as an educational tool" (WIKIPEDIA 2013). Gamification, on the other hand, is the use of game design elements in non-game contexts (SEBASTIAN, DAN, RILLA \& LENNART 2011). This definition is very broad to allow for the variety of gamification types but it creates a distinction between gamification and serious gaming. Similarly gamification is defined as the use of game thinking and game mechanics to engage users and solve problems (ZICHERMANN \& CUNNINGHAM 2011).

Applications where users create and share user generated content (UGC) are a common Web 2.0 theme. Most popular forms of UGC include text messaging, social media interactions, photos, video, blog entries, etc. UGC is essential for social networks (SN) as the two concepts mutually enhance each other. Social networks need to keep their users engaged in order to foster UGC creation. It is reported that more than 50\% of Facebook social network users have played a social network game (SNG) (LIGHTSPEED RESEARCH 2010).

The Location Based Services (LBS) industry has profited from UGC primarily because ubiquitous and affordable smartphones equipped with multiple sensors foster geographic data collection. Volunteered geographic information (VGI), as a special case of UGC, is the 
harnessing of tools to create, assemble, and disseminate geographic data provided voluntarily by individuals (GOODCHILD 2007). GOODCHILD also describes citizen sensing as network of human sensors that has over six billion components, each an intelligent synthesiser and interpreter of local information. General technological advances have also enabled a new paradigm that uses community (or crowd) participation to accomplish tasks, called crowdsourcing. Crowdsourcing distributes a task to a group of people, efficiently accomplishing a task which was traditionally accomplished by a few selected individuals.

Similarly, Location Based Social Networks (LBSN) leverage the power and high adoption rate of modern mobile devices to provide applications and services which allow users to share and discuss the real-world places they visit, as a part of their virtual interactions (FUREY, CURRAN \& KEVITT 2012). In terms of social networks location sharing has changed from a purpose-driven to social-driven activity. Users traditionally shared location with one other person (one-to-one) or with a small group (one-to-few). Social networks, depending on the privacy settings, enable users to share their location with a large group (oneto-many) or with everyone (one-to-all) (TANG, JiALIU, HONG, JASON, SIEWIOREK \& SADEH 2010).

Location Based games (LBG) are games in which the game play somehow evolves and progresses based on a player's location. Thus, LBG almost always support some kind of localisation technology, for example, by using satellite positioning like GPS, WiFi, Near Field Communication and Bluetooth. The blend of LBG and LBSN is a Location Based Social Network Game (LBSNG) exemplified by a service like Foursquare.

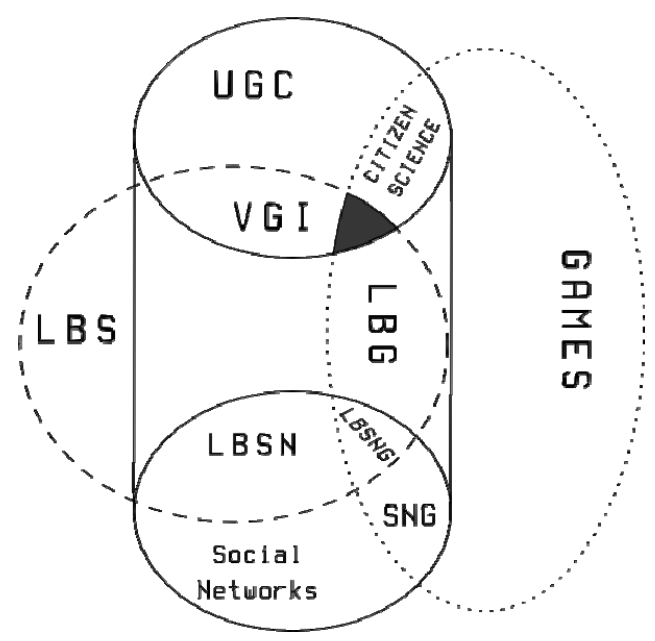

Fig. 1: Gamified geographic data collection with $\mathrm{UGC}=$ User Generated Content; VGI $=$ Volunteered Geographic Information; LBS = Location Based Services; LBG = Location Based Gaming; LBSN = Location Based Social Networks; SNG = Social Network Gaming; and LBSNG = Location Based Social Network Games

Citizen science is just one example of the amalgamation of UGC and gamification. Services like Zooniverse (ZOONIVERSE 2013) host numerous projects which allow users to partici- 
pate in scientific research from classifying galaxies to collating climate data. Projects use game elements to stimulate user participation.

In this review paper we focus on the intersection of VGI and gamification, as shown in Figure 1. The following section provides an overview of gamification and users' motivation for playing games. We also discuss basic game mechanics (game design) elements. Section three presents an analysis of several existing services that use game design elements in geographic data collection processes. We argue that gamification in geographic data collection processes will improve user engagement with the system. We conclude this paper by presenting our ongoing and future work.

\section{Gamification}

Games have a strong ability of imparting a sense of agency to the players, making them feel empowered and giving them the impression that their decisions are meaningful and will have an impact. A sense of agency refers to the subjective awareness that one is initiating, executing and controlling one's own volitional actions in the world (JEANNEROD 2003).

The most common game design element is a reward system. Rewards are divided into four distinct categories - status, access, power and stuff - the so-called SAPS model (ZICHERMANN \& CUNNINGHAM 2011). The four categories are ordered from most desired to least desired for the user and from the cheapest to the most expensive for the system designer:

- Status: the relative social position in relation to others. This is particularly important in social networks and LBSN.

- Access: rewards user by giving them early access to new features, promotions, sales etc.

- Power: partly transfers control to (other) users, e.g. a user is allowed to moderate content creation by other users.

- Stuff: a tangible reward or prize, e.g. a free drink.

\subsection{Motivation}

Thinking about player motivation is essential in game design, because without motivation a player will not be interested in progressing further within a game. Game designers aim to keep a player in a constant state of flow. Flow indicates the state in which a player is between anxiety and boredom and meeting their own motivational level in that experience (CSIKSZENTMIHALYI 1991).

Psychology has divided motivations into two groups: intrinsic and extrinsic (RYAN \& DECI 2000). Intrinsic motivations reflect a genuine interest in or enjoyment of an action for its own sake, reflecting the natural human ability to learn and assimilate. Extrinsic motivations are driven mostly by factors external to the agent (here: the user), e.g. money, fear of punishment etc.

Aggregation of meta-analytic psychology research (HECKER 2011) showed that for inherently interesting tasks most researchers agree on two points: 
- tangible, expected, contingent rewards reduce free-choice intrinsic motivation, while

- verbal, unexpected, informational feedback increases free-choice and self-reported intrinsic motivation.

Awarding tangible rewards (badges, points etc.) for an action will reduce this system to a means to an end. An action will be carried out for the sole purpose of achieving something else. In contrast, unexpected praise will motivate the player to play the game for its own sake.

\subsection{Game mechanics}

Game mechanics are a series of tools that promise to produce a meaningful response from players. There are seven primary elements: points, levels, leaderboards, badges, challenges/ quests, onboarding and engagement loops (ZICHERMANN \& CUNNINGHAM 2011).

Points enable a game designer to value and track every move and action a player makes in the game. Points are an elementary metric that enable game designers to make appropriate changes or adjustments depending on the respective outcome of (part of) a game. There are five basic point system designs which are briefly presented below:

- Experience - have no maximum; usually cannot be redeemed and do not expire; shows players' progress in the system.

- Redeemable - the basis for a virtual economy; exchangeable for rewards; can be used to tweak, optimize and manage the virtual economy of the game.

- Skill - bonus points assigned to specific activities within the game; used to steer player towards alternate tasks and sub-goals.

- Karma - the single purpose is to award points to others; similar to voting system.

- Reputation - purpose is to act as a proxy of trust; usually a complex aggregation of actions.

Levels are markers of progress as they serve as a marker for the player to know where they stand in the game over time. Levels can also indicate that the game has become more difficult and complex. The increase in difficulty is almost never linear, but in most cases curvilinear. However, rewards for desired behaviours always increase in value. A simple progress bar or more elaborate graphic representation is used to represent advancement to a new level or change of a level.

Leaderboard is an ordered list of names and scores, basically a ranking system. The traditional leaderboard display is a constantly updated list which can be a disincentive to a new player because the player starts at the very bottom of the list. A common improvement to leaderboard design is to show only part of the leaderboard which effectively always puts new players in the middle. Leaderboards can be also sliced depending on the context, e.g. locally (showing geographically close players), socially (showing friends and followers) and globally (based on any system metric).

Badges are used to define a successful end of an activity or a goal which indicates steady progress of a player's interaction with the system. Sometimes badges are used as a replacement of a level system. Badges are collectable items which are publicly displayed making them a kind of a social currency. 
In some systems badges are predefined making them only a means to an end. Once players collect a predefined badge they will lose interest in the activities which were required for that badge. In other systems distribution of badges includes randomness making them a pleasant surprise for the players. A good badge system will always be a balance of the predefined and random badges.

Challenges and quests are used to direct actions of a player within the system towards a long term (higher) goal. This game element can be optional, but it adds depth and meaning for the player. The basic idea is to provide the player with a constant challenge. Having a long term goal makes smaller repetitive activities less stressful.

Onboarding is a process of introducing a new player to the system. This process is all about maximising the new player's experience without making her/him feel overwhelmed and confused. Some systems are designed in a way that new players always win, maximising player experience.

In game design, engagement loops exploit players' social status in a way that players reengage in an activity in the system. Social networks are a good example as they have a strong user re-engagement loop which in essence pulls back in users which have left the system. This is usually executed by using a motivating emotion. For example, when someone mentions you on a social network, the system sends you a notification and uses particular methods to ensure you respond.

\section{Analysis of Gamified Location Services}

\subsection{Foursquare}

Foursquare describes itself as follows:

"a free app that helps you and your friends make the most of where you are. When you're out and about, use Foursquare to share and save the places you visit. And, when you're looking for inspiration for what to do next, we'll give you personalized recommendations and deals based on where you, your friends, and people with your tastes have been.” (FOURSQUARE 2013a)

Foursquare is the largest LBSN with nearly 30 million users of which more than four million check in every day.

Foursquare is a prime example of a gamified experience, as it uses every SAPS model element. Users receive status rewards by a combination of badges, leaderboards, levels and challenges. For example, if a user has checked in to a venue on more days than anyone else in the past 60 days, and the check-ins are valid under Foursquare's time and distance protocols, they will be crowned "mayor". Badges are public and as such are used as a social currency. Most badges are collected in a random fashion. However, there are challenges which promote certain desired activities, e.g. visiting ten coffee shops in a week. For every checkin users collect varied amount of points which are then used to create sliced leaderboards.

Power rewards are given by Foursquare in a form of a 'Super User' badge. Super users help to maintain the service by editing data about a location, add and merge categories; basically they act in a role of a community moderator. Access and stuff rewards are not directly 
facilitated by Foursquare, but by business which offer free stuff or discounts through checkins, often by repeated check-ins in the same business.

Even though geographic data is collected by users, and Foursquare has a rich Application Programming Interface (API), due to Foursquare's terms of use, the content can be used solely for personal, non-commercial use (FOURSQUARE 2013b). However, as Foursquare uses OpenStreetMap data to create its background "slippy" map, it is in their interest to cooperate with the OpenStreetMap community.

\subsection{Waze}

Waze is a free social mobile application that uses real-time input from motorists to update traffic conditions for other users by using GPS-enabled smartphones with a data plan. It differs from traditional GPS navigation software because it is a community-driven application that learns from users' driving times to provide routing and real-time traffic updates.

In terms of the SAPS model, Waze rewards users with status, power and stuff. While driving, Waze anonymously collects GPS data which is then used for real-time turn-by-turn navigation. Users can collect points for every meter of the road they drive on, by reporting accidents, traffic jams, speed traps and police or by editing data. Points are used to rank players on leaderboards which rewards players with status. Some users are rewarded with power in the sense that they are enabled to moderate community forums, monitor data changes and have grater editing privileges.

Waze also offers bonus points, which can be collected by driving over them. Bonus points are redeemable in form of gift certificates, lower gas prices etc. Bonus points are used to encourage more user involvement and competition, resulting in more valuable road information for the users and the database where those details are otherwise slim or lacking.

Even though Waze started as a GPL-licensed open source application, it is currently a proprietary application. However, data collected by users, was always proprietary. The Waze developers have a strong opinion against using OpenStreetMap data due to OSM data licensing terms which might limit the company's choice of business models in the future (WAZE 2013).

\subsection{Ingress}

Ingress is an augmented reality multiplayer online video game created by NianticLabs@ Google, which is available for Android devices. This game is also classified as an alternate reality game characterised by a narrative played out in the game. In order to play, players walk from place to place with Ingress running on their GPS-enabled smartphones and perform in-game tasks.

This game was silently released as an invite-only game on November 16, 2012, and because of that most details are still unknown. The game portrays an alternate reality setting in which two factions compete for control points. The winner of the game is the faction which controls the most "Mind units" enclosed by territory. In this context, "Mind units" represent the estimated number of humans within the territory controlled by the faction. The players have to be in the vicinity of a control point to perform actions. Relative to the SAPS model, the game rewards user with status as it uses points, levels and leaderboard game design elements. 


\subsection{Kort - an OpenStreetMap Game}

Kort is a web service optimised for mobile phones that combines the concept of gamification with volunteered geographic information and is targeted to non-specialists (ODERBOLZ \& HUNZIKER 2013). This game was developed by STEFAn ODERBOlZ and JÜRG HunZIKER as Bachelor Thesis in Computer Science at the University of Applied Sciences in Rapperswil, Switzerland (HuNZIKER \& ODERBOLZ 2012).

Players collect points (called Koins) by finishing missions. Examples of missions include adding names to points of interest, determining the language of an entry or definition of a speed limit on the road. Missions are updated on a daily basis by downloading problems from KeepRight service (http://keepright.at/). However, the service has not implemented direct modification of OpenStreetMap database. The process in which the result of a mission transforms into a proposed change requires validation by three or more users, depending on the mission type. Once a mission has been verified it needs to be manually uploaded to the OpenStreetMap database (http://www.kort.ch/proposals.php).

In this game players are only rewarded with status as the game implements points, leaderboards and badge game design elements. For example, a player gains five Koins for adding language of an entity, and for entering a missing name players are rewarded with 15 Koins. The badge system is simple as players are rewarded for the exact number of completed missions or validations, e.g. for ten missions a player gets the "ten completed missions" badge.

The game is an open source project (KORT 2013) and it can be enhanced and modified for any purpose. For example, adding new types of badges that are awarded in a more random pattern like "most completed missions per square kilometre". The project was developed with localisation in mind, so the interface can be easily translated into any language. As of January 2013, there are 13 fully supported languages.

\subsection{OpenStreetMap Reporter}

OpenStreetMap Reporter (http://osm.linfiniti.com/) started as a hobby project by TIM SUTTON in December 2012. The main idea was to sponsor three students to capture building footprints in Swellendam using available orthophoto satellite imagery (SUTTON 2012).

The service rewards users with status by ranking them on a leaderboard, and the user with the highest number of objects gets the "Guru" badge. The most interesting feature of the service is its leaderboard dynamicity. Leaderboards are sliced by geographical location which encourages competition between local users. Users can also choose between two types of reports, buildings and highways, which is a configurable option that depends on OpenStreetMap Overpass API supported data types.

The service is also an open source project written in Python using the Flask web application framework (OSM REPORTER 2013). Compared to other projects, the code base is really small which makes it the best candidate if someone wanting to start contributing to a project. For example, one of the next features could be integration with OpenStreetMap authentication service or historical contribution statistics for users. 


\section{Discussion}

In this paper we have given an overview of gamification and users motivation for playing games, and shown what basic game mechanics elements are. The review of several existing gamified geographic data collection services in terms of the SAPS model and used game design elements has shown that all services use points and leaderboards to reward users with status (Table 1). More expensive rewards are only available in services that are owned by companies.

Table 1: $\quad$ Reviewed services

\begin{tabular}{|l|l|l|l|l|l|}
\hline Service & Status & Access & Power & Stuff & Game elements \\
\hline Foursquare & Yes & Yes & Yes & Yes & $\begin{array}{l}\text { points, leaderboards, } \\
\text { badges, levels, challenges }\end{array}$ \\
\hline Waze & Yes & No & Yes & Yes & $\begin{array}{l}\text { points, leaderboards, } \\
\text { levels, challenges }\end{array}$ \\
\hline Ingress & Yes & No & No & No & points, leaderboards, challenges \\
\hline Kort & Yes & No & No & No & $\begin{array}{l}\text { points, leaderboards, } \\
\text { badges, challenges }\end{array}$ \\
\hline OSM Reporter & Yes & No & No & No & points, leaderboards \\
\hline
\end{tabular}

Gamification is not a simple panacea, one which is applicable to every problem in the same way. Use of gamification without any real context or integration in a thoughtful way with the underlying process will cause more problems than it tries to solve. For example, Kort service uses a system of predefined badges that reduce player activities to a means to an end. However, for currently implemented mission types and activities, a system of predefined badges could be a good enough motivator. In other contexts, predefined badges will have a negative effect on a system that deprives players of a positive gaming experience.

For example, GABE ZICHERMANN noted that "games are the only force in the known universe that can get people to take actions against their self-interest, in a predictable way, without using force" (ZICHERMANN 2010). If we use game design elements, on any system, we can improve actual user engagement with the system by making everything fun and pleasurable. However, gamification could also be considered as an exploitative series of shallow psychology tricks that enable owners of the systems to manipulate users of those systems into behaving the way the owners want.

LAZARRO's (2004) research on the factors that motivate people to play games found four main motivators: for mastery, to de-stress, to have fun and to socialize. Games create emotions in different aspects of the player experience. Emotions are also important for keeping players in the constant state of flow. Motivation is also one of the factors that keep players in the constant state of flow. The current iteration of gamified services is mainly focused on the exploitation of extrinsic motivators. This demands that a gamified experience provides a constant stream of rewards. Users will simply lose interest and disengage from the system if they have already achieved all of the available rewards or if rewards are turned off. This effect is known as "overjustification", where initial intrinsic motivation is shifted towards extrinsic incentives (LEPPER, GREENE \& NISBETT 1973). This means that service providers need to invest more resources into keeping the system running. 
Large corporations, like Google and Foursquare, have already created a business around gamified geographic data collection. The fact that the user is collecting data is masked by keeping user in constant state of flow, immersed deeply in the game. Therefore, geographic data collection should be a secondary by-product of a user's play experience.

The appeal of the Ingress game to its users enables Google to use this game as a data collection service. For instance, blog writer DEREK GILDEA, notes that "Google is cajoling its users to provide it with real-world-data on how best to get from point A to B on foot" (GILDEA 2012). Conveniently, Ingress fits perfectly into Google's data collection platform, as it is collecting data which cannot be extracted from StreetView or GoogleEarth projects.

The largest VGI project, OpenStreetMap, continues to grow in every aspect. Research by MASHHADI, QUATTRONE, CAPRA \& MOONEY (2012) shows that for urban areas, point-ofinterest (POI) OpenStreetMap data is highly accurate, with accuracy defined in terms of geographic (positioning) error, lexicographic error and amenity error (MASHHADI et al. 2012). However, only a small number of users, called power users, contribute most of the content. A large majority of users only contributes some content changes.

With regard to the intersection of gamification and VGI, only two of the analysed services have direct a relation to OpenStreetMap, Kort and OSM Reporter. OpenStreetMap project contributors are almost exclusively intrinsically motivated. Contributors are rewarded with status which is not artificial, but accumulated by direct actions in a meritocratic community. The addition of extrinsic motivators to the process of VGI collection could improve overall contributor engagement within the system.

\section{Conclusion and Future Work}

Gamification can be used to promote desired activities. This is useful when organising a distributed geographic data collection effort. We plan to focus our further research on specific game elements in the context of geographic data collection, particularly in the realm of volunteered geographic information and existing open source projects. We will also prepare a proposition for the implementation of a reusable service platform that will facilitate gamified VGI collection. This platform will serve as a test bed for gamified geographic data collection.

\section{References}

CSIKSZENTMIHALYI, M. (1991), Flow: The psychology of optimal experience: Steps toward enhancing the quality of life. Harper Collins Publishers.

Deterding, S., Dixon, D., Khaled, R. \& NACKe, L. (2011), From Game Design Elements to Gamefulness: Defining “Gamification”. Proceedings of MindTrek.

FOURSQUARE (2013a), About Foursquare. https://foursquare.com/about/ (January 16, 2013).

FOURSQUARE (2013b), Foursquare Labs, Inc. Terms of Use. https://foursquare.com/legal/terms/ (January 15, 2013).

Furey, E., Curran, K. \& KevitT, P. (2012). Probabilistic indoor human movement modeling to aid first responders. Journal of Ambient Intelligence and Humanized Computing, 1-11. doi:10.1007/s12652-012-0112-4. 
GILDEA, D. (2012), Very clever: Google's Ingress masks Data-Collection in Gaming http://takefiveblog.org/2012/12/09/ingress-gathering-data-through-gaming/ (January 5, 2013).

GoodchiLd, M. F. (2007), Citizens as sensors: the world of volunteered geography. GeoJournal, 69 (4), 211-221.

HeCKer, C. (2011), Achievements Considered Harmful? Page last updated on March 6, 2011.http://chrishecker.com/Achievements_Considered_Harmful\%3F

(November 20, 2012).

HunziKer, J. \& Oderbolz, S. (2012), Gamified Mobile App für die Verbesserung von OpenStreetMap. Rapperswil: Abteilung Informatik, HSR Hochschule für Technik.

JEANNEROD, M. (2003), The mechanism of self-recognition in humans. Behavioural Brain Research, 142 (1-2), 1-15.

KORT (2013), Kort code repository. https://github.com/odi86/kort (January 3, 2013).

LAzZaro, N. (2004), Why We Play Games: Four Keys to More Emotion Without Story. Game Developers Conference.

LePPER, M. P., GReENe, D. \& NisbetT, R. E (1973), Undermining children's Intrinsic interest with extrinsic reward: A test of the "overjustification" hypothesis. Journal of Personality and Social Psychology, 28 (1), 129-137.

LIGHTSPEED RESEARCH (2010), It's game on for Facebook users. http://www.lightspeedresearch.com/press-releases/it $\% \mathrm{E} 2 \% 80 \% 99$ s-game-on-forfacebook-users/ (December 26, 2012).

Mashhadi, A., Quattrone, G., CaPra, L. \& MoOney, P. (2012), On the Accuracy of Urban Crowd-Sourcing for Maintaining Large-Scale Geospatial Databases. WikiSym.

Oderbolz, S. \& HunZIKER, J. (2013), Kort - an OpenStreetMap Game. http://www.kort.ch/ (January 13, 2013).

OSM REPORTER (2013), OpenStreetMap code repository. https://github.com/timlinux/osmreporter (January 23, 2013).

RYAN, R. M. \& DECI, E. L. (2000), Intrinsic and Extrinsic Motivations: Classic Definitions and New Directions. Contemporary Educational Psychology, 25 (1), 54-67.

Sebastian, D., Dan, D., Rilla, K. \& Lennart, N. (2011), From Game Design Elements to Gamefulness: Defining “Gamification”. Proceedings of MindTrek.

SuTTON, T. (2012), Holiday OpenStreetMap project for Swellendam. http://linfiniti.com/2012/12/holiday-openstreetmap-project-for-swellendam/ (December 17, 2012).

Tang, K. P., Jialiu, L., Hong, L., Jason, I., Siewiorek, D. P. \& Sadeh, N. (2010), Rethinking location sharing: exploring the implications of social-driven vs. purposedriven location sharing. Proceedings of the $12^{\text {th }}$ ACM international conference on Ubiquitous computing, 85-94. Denmark, ACM.

WAZE (2013), Waze: Frequently Asked Questions. http://www.waze.com/faq/ (Jaunary 17, 2013).

WIKIPEDIA (2013), Game.

https://en.wikipedia.org/w/index.php?title=Game\&oldid=533542403 (January 23, 2013).

ZichermanN, G. (2010), Fun is the Future: Mastering Gamification, Google TechTalk. https://www.youtube.com/watch? $\mathrm{v}=6 \mathrm{O} 1 \mathrm{gNVeaE} 4 \mathrm{~g}$ (January 12, 2013).

ZichermanN, G. \& CUnNinghaM, C. (2011). Gamification by Design: Implementing Game Mechanics in Web and Mobile Apps. O'Reilly Media, Inc.

ZOONIVERSE (2013), Zooniverse. https://www.zooniverse.org/ (January 15, 2013). 